\section{Uniaxial pressure control of competing orders in a high-temperature superconductor}

\author{
H.-H. Kim ${ }^{1 *}$, S. M. Souliou ${ }^{2 *}$ †, M. E. Barber ${ }^{3}$, E. Lefrançois ${ }^{1,2}$, M. Minola ${ }^{1}$,

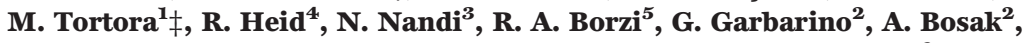

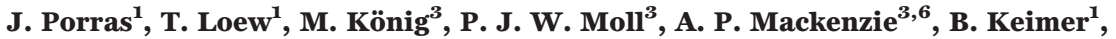 \\ C. W. Hicks ${ }^{3}$, M. Le Tacon ${ }^{4} \S$
}

Cuprates exhibit antiferromagnetic, charge density wave (CDW), and high-temperature superconducting ground states that can be tuned by means of doping and external magnetic fields. However, disorder generated by these tuning methods complicates the interpretation of such experiments. Here, we report a high-resolution inelastic x-ray scattering study of the high-temperature superconductor $\mathrm{YBa}_{2} \mathrm{Cu}_{3} \mathrm{O}_{6.67}$ under uniaxial stress, and we show that a three-dimensional long-range-ordered CDW state can be induced through pressure along the a axis, in the absence of magnetic fields. A pronounced softening of an optical phonon mode is associated with the CDW transition. The amplitude of the CDW is suppressed below the superconducting transition temperature, indicating competition with superconductivity. The results provide insights into the normal-state properties of cuprates and illustrate the potential of uniaxial-pressure control of competing orders in quantum materials.

M oderately doped high-temperature superconductors show an ubiquitous tendency toward charge order (I). Manifestations of charge ordering include striped order-an incommensurate modulation of both charge and spin that suppresses superconductivity in lanthanum-based cuprates $(2,3)$ - and a biaxial charge density wave (CDW) with quasi-two-dimensional (2D) short-range order in the $\mathrm{CuO}_{2}$ planes of all other cuprate families (4-12). The origin of CDW order and its relationship with superconductivity are widely debated issues. It is well-established that static CDW order and superconductivity compete; however, it is not clear whether the two orders are best described as mutually incompatible $(7,8)$, as different manifestations of the same pairing interaction (13), or as dif-

\footnotetext{
${ }^{1}$ Max Planck Institute for Solid State Research, Heisenbergstraße 1 D-70569 Stuttgart, Germany. ${ }^{2}$ European Synchrotron Radiation Facility (ESRF), BP 220, F-38043 Grenoble Cedex, France. ${ }^{3}$ Max Planck Institute for Chemical Physics of Solids, Nöthnitzer Straße 40, 01187 Dresden, Germany. ${ }^{4}$ Institute for Solid State Physics, Karlsruhe Institute of Technology. Hermann-v.-Helmholtz-Platz 176344 Karlsruhe, Germany. ${ }^{5}$ Instituto de Física de Líquidos y Sistemas Biológicos (IFLYSIB), UNLP-Consejo Nacional de Investigaciones Científicas y Técnicas (CONICET), La Plata, Argentina and Departamento de Física, Facultad de Ciencias Exactas, Universidad Nacional de La Plata (UNLP), c.c. 16, suc. 4, 1900 La Plata, Argentina. ${ }^{6}$ Scottish Universities Physics Alliance, School of Physics and Astronomy, University of St Andrews, St Andrews KY16 9SS, UK

*These authors contributed equally to this work.

†Present address: Institute for Solid State Physics, Karlsruhe Institute of Technology, Hermann-v.-Helmholtz-Platz 176344 Karlsruhe, Germany.

†Present address: ams AG, Tobelbader Straße 30, 8141

Premstätten, Austria.
}

§Corresponding author. Email: matthieu.letacon@kit.edu ferent aspects of a composite order parameter $(14,15)$. These issues have direct and important implications for the mechanism of hightemperature superconductivity (HTSC).

The $\mathrm{YBa}_{2} \mathrm{Cu}_{3} \mathrm{O}_{6+x}\left(\mathrm{YBCO}_{6+x}\right)$ family has been particularly well studied because dopinginduced structural disorder is less severe than in other families. In this compound, the competition between superconductivity and CDW order is evidenced by the depression of the CDW amplitude upon cooling below the superconducting transition temperature $\left(T_{\mathrm{c}}\right)$ $(7,8)$ and by its enhancement in magnetic fields that weaken superconductivity $(8,9)$. Nuclear magnetic resonance (NMR) and $\mathrm{x}$-ray studies further showed that in fields larger than $\sim 15 \mathrm{~T}$, a 3D long-range ordered uniaxial CDW (16-19) is induced. This 3D order is distinct from the $2 \mathrm{D}$ one, although they coexist and are related to each other; for example, they have the same in-plane incommensurability. The 3D order has an identifiable thermodynamic transition (20-22), whereas the 2D CDW onsets gradually $(23,24)$, and it is not clear whether it constitutes an vestige of the $3 \mathrm{D}$ order that is weakened by the interaction with superconductivity.

We used inelastic $\mathrm{x}$-ray scattering (IXS) on a sample of $\mathrm{YBCO}_{6.67}\left(T_{\mathrm{c}}=65 \mathrm{~K}\right.$, doping $p=$ 0.12 ) to show that uniaxial pressure along the crystallographic $a$ axis can induce longrange 3D CDW order, in the absence of a magnetic field.

We first specify why we chose to work with uniaxial pressures. In the doping range of interest $(0.08<p<0.15)$, hydrostatic pressure yields, through self-doping and other effects, alternative ground state or is, for example, a an increase of $T_{\mathrm{c}}(25)$. A recent study of the biaxial charge modulation further revealed its rapid suppression under hydrostatic pressure (26). However, it has long been established that, at least in the limit of low pressures, the effect of hydrostatic pressure on $T_{\mathrm{c}}$ reflects a net sum of larger effects of uniaxial pressures that almost cancel each other out $(27,28)$. In particular, close to $p \sim 0.12$, where the charge modulation is maximized, uniaxial pressure applied along the $a$ axis suppresses $T_{\mathrm{c}}$ $(29,30)$.

In this study, we pressurized the sample using a piezoelectric-based apparatus similar to that used in recent studies of ruthenium oxides (31-33) but modified to allow x-ray transmission through the apparatus and the sample. For maximum scattering intensity, the thickness of the sample should match the absorption length at the working wavelength $(\lambda=0.6968 \AA$ for this experiment), which is $\sim 40 \mu \mathrm{m}$. However, to withstand strong compression without buckling, the lengthto-thickness ratio of the sample cannot be too large (31), and a 40- $\mu \mathrm{m}$ thickness implies a length that is inconveniently short for reliable mounting. Therefore, we prepared a thicker needle from a $\mathrm{YBCO}_{6.67}$ single crystal then used a plasma focused ion beam (PFIB) to thin down to $40-\mu \mathrm{m}$ thickness the central portion over a length of $\sim 200 \mu \mathrm{m}$ (Fig. 1). The x-ray beam, with a spot size of $50 \times 40 \mu \mathrm{m}^{2}$, was considerably smaller than the thinned central portion of the sample, so highly uniform strain is expected in the probed volume. All the strain values in this paper were calculated by using capacitive displacement sensor built into the pressure cell, the changes in the $c$ axis lattice parameter measured from the (l $\left.\begin{array}{lll}0 & 0\end{array}\right)$ Bragg peak and the changes in $T_{\mathrm{c}}$ (30). The highest compression we reached was $\varepsilon_{x x} \sim-1.0 \%$, where $a c$ susceptibility measurements showed that $T_{\mathrm{c}}$ decreases (at a rate increasing with strain) to $48 \pm 5 \mathrm{~K}$ (30).

The data on the 2D biaxial CDW are shown in Fig. 2. The scattering intensity of the biaxial CDW peaks at the reduced momentum transfer $\boldsymbol{q}_{2 \mathrm{D}}=(h, k, l)=(0,0.31,0.5)$, in reciprocal lattice units (r.l.u.) of the orthorhombic crystal structure $(7,8)$. By contrast, the 3D CDW peaks at $\boldsymbol{q}_{3 \mathrm{D}}=(0,0.31,1)(16-19)$. Here, we will mostly refer to the total momentum transfers $\boldsymbol{Q}_{2 \mathrm{D}}=$ $(\mathrm{H}, \mathrm{K}, \mathrm{L})=(0,0.31,6.5)$ and $\boldsymbol{Q}_{3 \mathrm{D}}=(0,0.31,7)$, around which measurements were carried out and where the structure factor of the CDWs is maximum $(34,35)$. The color maps in Fig. 2, A and $\mathrm{B}$, show the raw IXS intensity at $\varepsilon_{x x}=0 \%$ (Fig. 2A) and at $-1.0 \%$ (Fig. 2B). Both data sets were measured below $T_{\mathrm{c}}(30)$. Two characteristic features of the CDW are clearly visible: the quasi-elastic "central" peak at $\boldsymbol{Q}_{2 \mathrm{D}}$ and the superconductivity-induced Kohn anomaly in the phonon spectra. In unstrained conditions, the latter consists of a $\sim 15 \%$ softening of the low-lying acoustical phonon (at $\sim 8 \mathrm{meV}$ ) at $\boldsymbol{Q}_{2 \mathrm{D}}$ (35). Comparing Fig. 2, A and B, it can be seen that the quasi-elastic peak is enhanced by the 
applied pressure. In Fig. 2C, it is seen that this enhancement occurs smoothly. At the highest strain, the integrated intensity of the peak is close to two times larger than that of the unstrained sample (Fig. 2D). Its half-widthat-half-maximum (HWHM) $\sigma$, which is inversely proportional to the modulation correlation length $\xi=\frac{1}{2 \pi \sigma}$, decreases modestly under pressure (Fig. 2D). We did not resolve any shift of $\boldsymbol{Q}_{2 \mathrm{D}}$ with $\varepsilon_{x x}$. Last, a comparison of
Fig. 2, A and B, reveals an increase of the low-energy spectral weight under pressure. Its phenomenology and relation to the Kohn anomaly will be discussed below.

We observed a much more notable response to uniaxial pressure in the scattering pattern at $\boldsymbol{Q}_{3 \mathrm{D}}$. We first looked at the strain dependence of the elastic peak intensity along the $\boldsymbol{Q}=(0,0.315, L)$ line at $T=50 \mathrm{~K}$ (Fig. 3A). At $\varepsilon_{x x} \sim-0.8 \%$, a small, narrow peak appears at $\boldsymbol{Q}_{3 \mathrm{D}}$. When the compression is further increased, to $\varepsilon_{x x} \sim-1.0 \%$, this peak becomes much more intense. It appears on top of the broad profile centered around $l=0.5(L=$ $6.5)$ that arises from the $2 \mathrm{D} \mathrm{CDW}$. The profile of the 3D peak along $K$ is shown on Fig. 3B. A weak 3D peak is visible at compressions as low as $\varepsilon_{x x} \sim-0.5 \%$; however, the increase in intensity from 0.8 to $1.0 \%$ compression dwarfs the evolution at lower compressions.
A

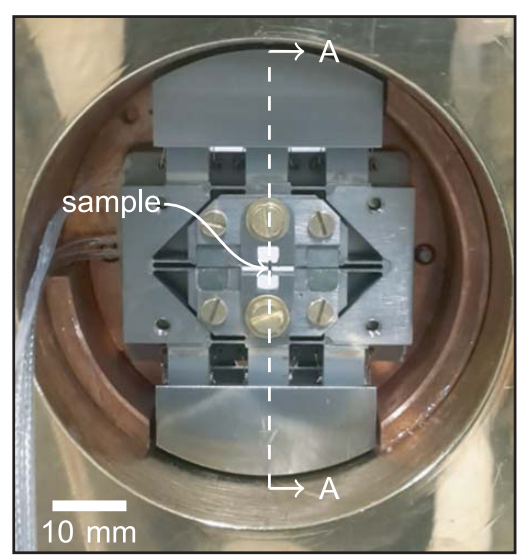

B

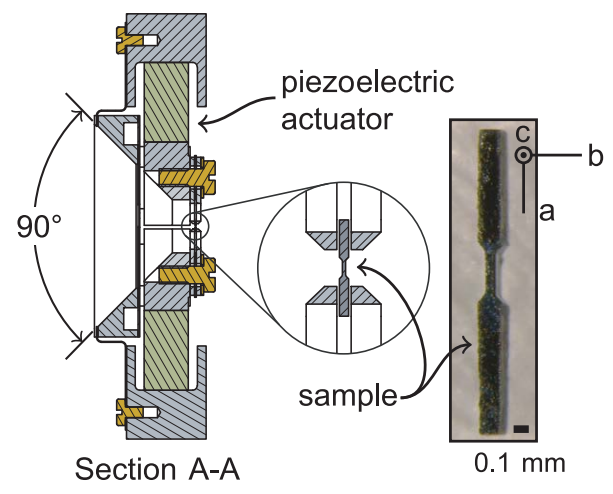

C

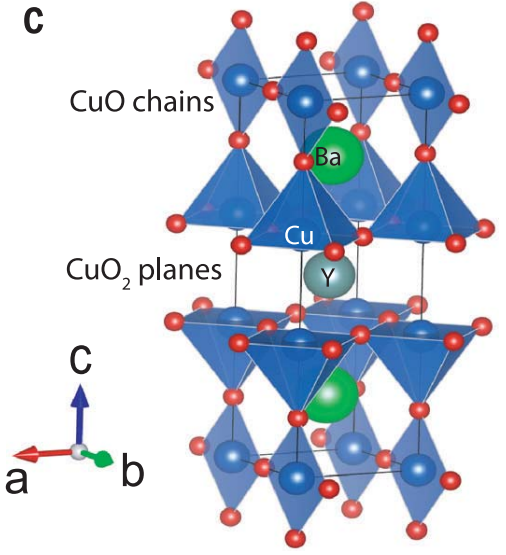

Fig. 1. Strain device. (A) Photograph of the piezoelectric device. (B) Sectional cut of the device and picture of the PFIB-thinned sample used for this experiment. (C) Unit cell of $\mathrm{YBa}_{2} \mathrm{Cu}_{3} \mathrm{O}_{6+x}$ (here with $x=1$ ). Strain is applied perpendicular to the $\mathrm{CuO}$ chains.

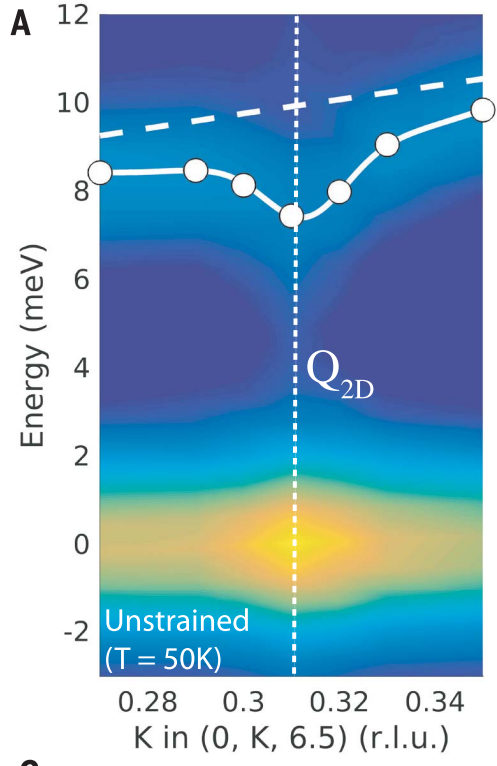

C

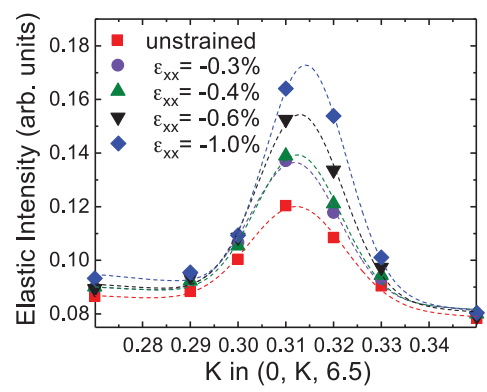

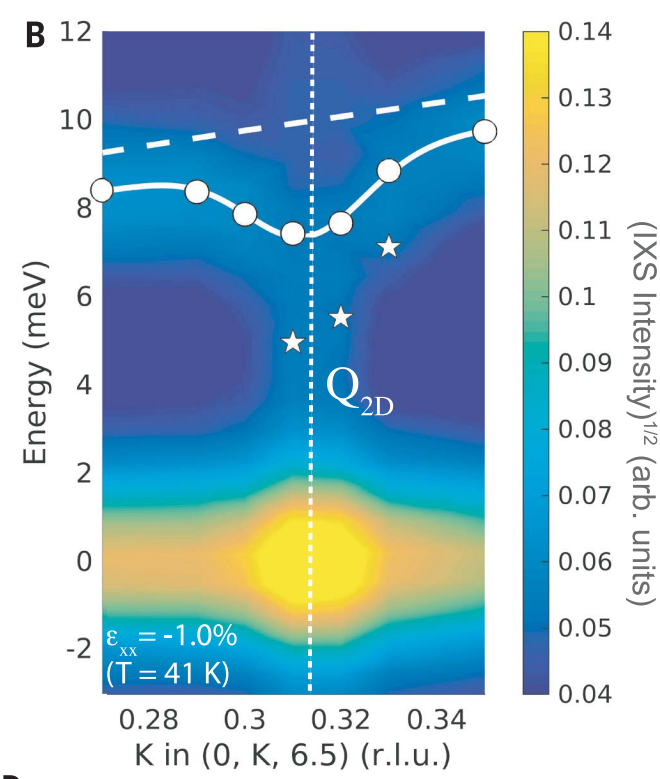

D

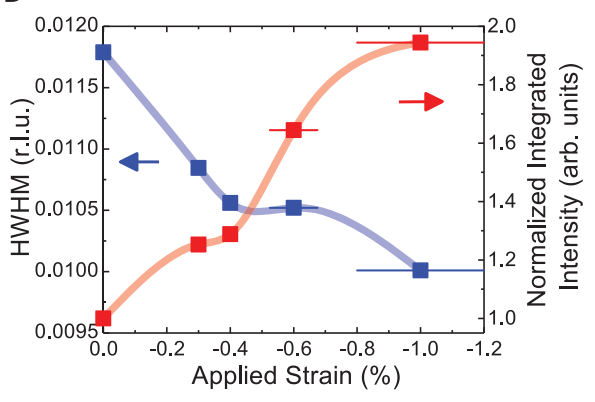

Fig. 2. Strain dependence of the IXS spectra around $\mathbf{Q}_{2 \mathrm{D}}$. (A) IXS intensity versus total momentum transfer for the unstrained sample at $T<T_{\mathrm{c}}$. The square root of the intensity has been plotted to enhance the contrast between the phonon and the elastic line. The calculated dispersion of the low-lying acoustical mode is plotted as a dashed line. The solid line is a guide to the eye to the observed dispersion of this mode (fitted values are represented by the dots). (B) Same as in (A), but for $\varepsilon_{x x} \sim-1.0 \%$. The stars indicate the energy of a soft optical phonon. (C) Strain-dependence of the quasi-elastic line intensity across $\mathbf{Q}_{2 \mathrm{D}}$ along the $(0, K, 6.5)$ direction. (D) Strain dependence of the HWHM and of the integrated intensity (normalized to the unstrained value) of the quasi-elastic line at $\mathbf{Q}_{2 \mathrm{D}}$, integrated along $K$ and normalized to the unstrained value. 

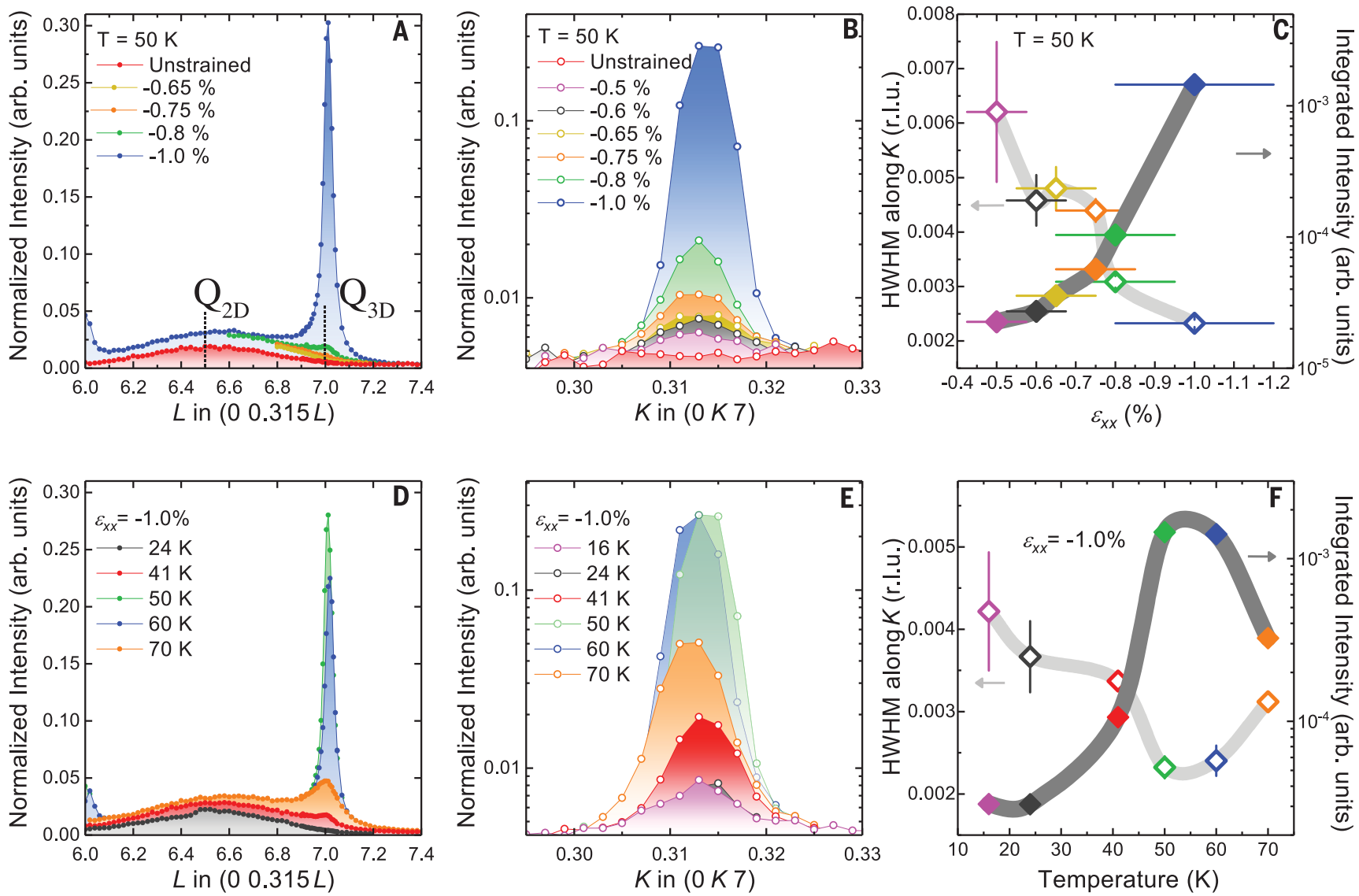

Fig. 3. Strain dependence of the quasi-elastic line at $\mathbf{Q}_{\text {3D. }}(\mathbf{A})$ Quasi-elastic intensity at $T=50 \mathrm{~K}$ along the $(0,0.315, L)$ direction as function of a axis compression $\varepsilon_{x x}$. (B) Quasi-elastic intensity at $T=50 \mathrm{~K}$ along the $(0, K, 7)$ direction as function of a axis compression $\varepsilon_{x x}$. (C) Strain dependence of the HWHM and the integrated intensity along the $(0, K, 7)$ direction at $T=50 \mathrm{~K}$. (D) Temperature dependence of the quasi-elastic intensity along the $(0,0.315, L)$ direction for $\varepsilon_{x x} \sim-1.0 \%$. (E) Temperature dependence of the quasi-elastic intensity along the $(0, K, 7)$ direction for $\varepsilon_{x x} \sim-1.0 \%$. (F) $H W H M$ and integrated intensities versus temperature at $\mathbf{Q}_{3 D}$ for $\varepsilon_{x x} \sim-1.0 \%$.

The evolution of the integrated intensity, and the HWHM along $K$, are shown in Fig. 3C. At $\varepsilon_{x x} \sim-1.0 \%$, the HWHM is $\sigma_{b} \sim 0.002$ r.l.u. It is resolution-limited along $L\left(\sigma_{c} \sim 0.02\right.$ r.l.u.). These correspond to respective (lower bounds for the) correlation lengths of $\xi_{b}=\frac{1}{2 \pi \sigma_{b}} \sim$ $80 b \sim 310 \AA$ and $\xi_{c}=\frac{1}{2 \pi \sigma_{c}} \sim 8 c \sim 94 \AA$ (the limitations of the scattering geometry did not permit investigation of $\xi_{a}$ ). These correlation lengths are larger than the values reported under a field of $26 \mathrm{~T}$. The correlation lengths of the $2 \mathrm{D}$ CDW at $1.0 \%$ compression are $\xi_{b}^{2 \mathrm{D}} \sim$ $16 b \sim 65 \AA$ and $\xi_{c}{ }^{2 \mathrm{D}} \sim c \sim 12 \AA$. To estimate the correlation volume $\Xi=\xi_{a} \times \xi_{b} \times \xi_{c}$, we estimate $\xi_{a} \sim \xi_{b}$ for both the 2D and 3D CDWs. For the 3D order, we find $\Xi \sim 51,000$ unit cells under uniaxial pressure for $T \sim T_{\mathrm{c}}$, exceeding by more than two orders of magnitude that of the 2D CDW at ambient conditions ( $\Xi \sim 250$ unit cells).

The temperature evolution of the 3D CDW at $\varepsilon_{x x} \sim-1.0 \%$ is shown in Fig. 3, D to F. The peak is very strong at $60 \mathrm{~K}$ and weaker but still visible at $70 \mathrm{~K}$. That is higher than the onset temperature of the $3 \mathrm{D}$ order observed under high field and higher than $T_{\mathrm{c}}$ of unstressed sample. On the low-temperature side, strong competition with superconductivity is apparent. At $T=41 \mathrm{~K}$ (below $T_{\mathrm{c}}$ ), the peak at $\boldsymbol{Q}_{3 \mathrm{D}}$ has already lost $\sim 90 \%$ of the integrated intensity recorded at $50 \mathrm{~K}$ and can hardly be distinguished from the background of the $2 \mathrm{D}$ order at lower temperatures. This is a much more rapid suppression than that seen for the 2D CDW (7).

To gain further insight on the relationship between the $2 \mathrm{D}$ and $3 \mathrm{D}$ orders, we investigated the pressure and temperature dependence of the phonon modes in the inelastic part of the spectra. In Fig. 4A, we show the phonon spectra in the absence of applied pressure at $T=$ $50 \mathrm{~K}$, along the $(0, K, 7)$ direction. Well away from $\boldsymbol{Q}_{3 \mathrm{D}}$, three peaks are visible. These are well reproduced in ab initio lattice dynamics calculations (30) and correspond respectively to an acoustic phonon mode of the $\Delta_{4}^{\prime}$ irreducible representation (36) at $\sim 8 \mathrm{meV}$, two optical modes $\left(\Delta^{\prime}{ }_{1}\right.$ and $\left.\Delta_{4}^{\prime}{ }_{4}\right)$ at $\sim 11 \mathrm{meV}$ (which are not distinguishable in the measurement), and another $\Delta_{1}^{\prime}$ optical mode at $\sim 15 \mathrm{meV}$. It can be seen that in the absence of strain, the acoustic-mode softening associated with the 2D CDW (34), and shown at $\boldsymbol{Q}_{2 \mathrm{D}}$ in Fig. 2A, extends along $L$ and is visible at $\boldsymbol{Q}_{3 \mathrm{D}}$.
In Fig. 4B, we show the same spectra but with $\varepsilon_{x x} \sim-1.0 \%$ and $T=41 \mathrm{~K}$ (to stay below $T_{\mathrm{c}}$ ). Away from $\boldsymbol{Q}_{3 \mathrm{D}}$, the spectra are essentially unchanged. Near $\boldsymbol{Q}_{3 \mathrm{D}}$, on the other hand, a very strong phonon softening is observed, albeit not of the acoustic mode, which now disperses exactly as predicted by the ab initio calculations as $K$ is swept through $\boldsymbol{Q}_{3 \mathrm{D}}$. In other words, the Kohn anomaly seen in Fig. 4A, at $T=50 \mathrm{~K}$ and $\varepsilon_{x x} \sim$ $0 \%$, is no longer present under $\varepsilon_{x x} \sim-1.0 \%$. This suppression of the acoustical Kohn anomaly can also be seen in Fig. 4C, where we show the $L$-dependence of the phonon spectra from $\boldsymbol{Q}_{2 \mathrm{D}}$ to $\boldsymbol{Q}_{3 \mathrm{D}}$ for both $\varepsilon_{x x} \sim 0 \%$ and $\varepsilon_{x x} \sim-1.0 \%$. Without pressure, the acoustical phonon is soft along the entire $L$-line, which is in agreement with the data in Fig. 4A. At $\varepsilon_{x x} \sim-1.0 \%$, we can follow the hardening of the acoustical modethe disappearance of the Kohn anomaly-as we traverse from $\boldsymbol{Q}_{2 \mathrm{D}}$ to $\boldsymbol{Q}_{3 \mathrm{D}}$. The mode that softens approaching $\boldsymbol{Q}_{3 \mathrm{D}}$ is a distinct feature, which we therefore identify as one of the optical modes. The temperature dependence of the phonon modes at $\boldsymbol{Q}_{3 \mathrm{D}}$ is shown in Fig. 4, D and $\mathrm{E}$. At $70 \mathrm{~K}$, the optical phonon is already very soft, which indicates that, unlike the acoustical Kohn anomaly, the optical mode softening 


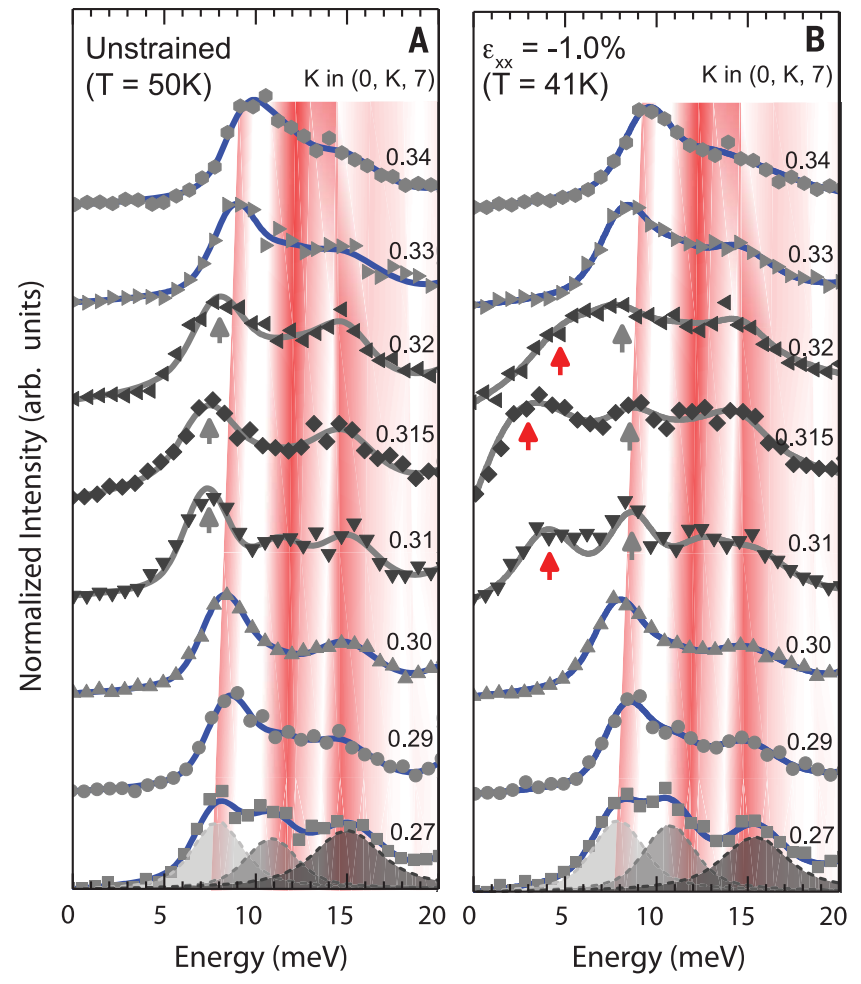

Fig. 4. Strain dependence of the phonon spectra around $Q_{3 D}$. $(A) I X S$ intensity in the absence of strain recorded below $T_{c}$ (at $T=50 \mathrm{~K}$ ) along the $(0, K, 7)$ direction. The elastic line has been subtracted (30). (B) Same for $\varepsilon_{x x} \sim-1.0 \%$, and, to remain below $T_{c}$, at $T=41 \mathrm{~K} . \ln (\mathrm{A})$ and $(\mathrm{B})$, the red lines represent the calculated structure factor of the phonons (30), the gray arrows indicate the acoustical phonon, and the red arrows indicate the soft optical mode. (C) $L$-dependence of the inelastic spectra. Open symbols indicate $T=50 \mathrm{~K}, \varepsilon_{x x} \sim 0$; closed symbols indicate $T=41 \mathrm{~K}$,
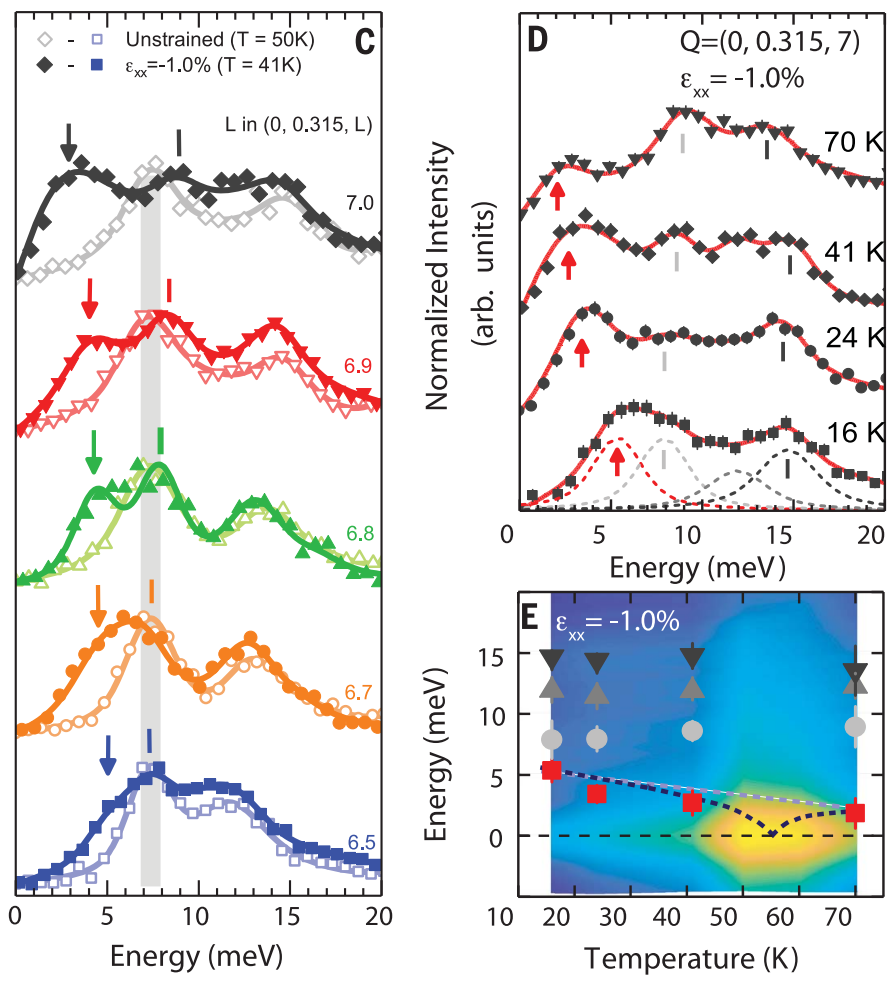

$\varepsilon_{x x}-1.0 \%$. For the strained data, the ticks indicate the energy of the acoustical mode, and the arrows indicate the energy of the soft optical phonon. (D) Temperature dependence of the phonon spectra at $\mathbf{Q}_{3 \mathrm{D}}$ for $\varepsilon_{x x} \sim-1.0 \%$. Solid lines in (A) to (D) correspond to the least square fitting of the data (30). (E) Phonon energy versus temperature superimposed to the IXS intensity color map (log scale) at $\mathbf{Q}_{3 \mathrm{D}}$ for $\varepsilon_{x x} \sim-1.0 \%$. Dotted lines are guide to the eyes for possible scenarios for the temperature dependence of the soft-phonon energy. is not induced by superconductivity. At 50 and $60 \mathrm{~K}$, the soft phonon mode cannot be resolved from the strong elastic peak (30). The mode hardens below $50 \mathrm{~K}$, as the long-range $\mathrm{CDW}$ order disappears in the superconducting state. Although a continuous hardening of the softphonon upon cooling through $T_{\mathrm{c}}$ cannot be completely ruled out with the data at hand (Fig. 4E), this behavior suggests a complete softening of this mode at Q3D, in analogy with soft-node-driven CDW orders [such as in 2H.-NbSe2 (37)].

The unambiguous identification of the soft phonon among the 36 allowed optical modes (38) is not a trivial task. Both $\Delta_{1}^{\prime}$ and $\Delta_{4}^{\prime}$ representations include an oxygen bond-stretching phonon around $60 \mathrm{meV}(39)$ that exhibits pronounced dispersion anomalies close to $\boldsymbol{Q}_{3 \mathrm{D}}(40)$. The buckling mode $\left(\Delta_{1}^{\prime}\right)$ also softens anisotropically along the 010 direction upon entering the superconducting state (41). Various scenarios have been discussed, attributing these anomalies to, for example, coupling of the phonons to dispersive collective charge excitations $(42,43)$ or to hybridization with lower energy branches of the same symmetry (44).

Further work is required to determine which of these optical modes is driven soft under uni- axial pressure, to determine whether the softening is complete and to understand the mechanism yielding the disappearance of the acoustical phonon Kohn anomaly at $\boldsymbol{Q}_{3 \mathrm{D}}$. The absence of phonon softening in single-electron calculations indicates that electronic correlations need to be included in any theoretical treatment of the phonon softening and CDW formation (45).

From a theoretical point of view, unidirectional CDW are unstable against disorder, and a vestigial nematic state is expected instead. It has previously been discussed how short-range biaxial modulation of the charge density might emerge from an intrinsic unidirectional CDWs instability in the presence of quenched disorder $(23,46,47)$, which locally reorients small uniaxial domains. Inhomogeneous distribution of the disorder strength has been invoked (18) to explain why the 2D order appears to strengthen under large magnetic fields even as unidirectional 3D order appears. Although one might expect the 3D order to grow at the expense of the 2D one, in this model this process occurs on top of a general strengthening of CDW order as superconductivity is suppressed by the magnetic field, and inhomogeneity in the strength of disorder allows spatially separated, coexisting domains of $2 \mathrm{D}$ and $3 \mathrm{D}$ order.
Similarly, our data indicate that strain-tuning efficiently strengthens the CDW and supports the formation of the 3D order, likely primarily in those regions where the pinning strength is the weakest. It will be interesting to see in the future whether the strength of the $2 \mathrm{D}$ order does eventually decrease as strain is further increased. More generally, further theoretical work is required to understand the strain-induced strengthening of the CDW, which cannot be solely attributed to the competition with superconductivity because the 3D CDW peak can already be induced above the nominal $T_{\mathrm{c}}$ of the sample.

Uniaxial stress will allow the relationship between the superconductivity and CDW to be investigated with high precision in future experiments. A magnetic field suppresses type II superconductivity inhomogeneously because of the presence of vortices, resulting in broad transitions. By contrast, the homogeneous tuning provided by stress could, for example, allow use of thermodynamic probes to determine whether CDW and superconductivity can coexist microscopically. Our piezoelectric-based apparatus constitutes a versatile tool that can be implemented in a large variety of experimental setups-in particular, at synchrotron facilities-thus opening 
perspectives for the study of correlated-electron materials.

\section{REFERENCES AND NOTES}

1. B. Keimer, S. A. Kivelson, M. R. Norman, S. Uchida, J. Zaanen, Nature 518, 179-186 (2015)

2. J. M. Tranquada, B. J. Sternlieb, J. D. Axe, Y. Nakamura, S. Uchida, Nature 375, 561-563 (1995)

3. M. Vojta, Adv. Phys. 58, 699-820 (2009)

4. J. E. Hoffman et al., Science 295, 466-469 (2002).

5. C. Howald, H. Eisaki, N. Kaneko, M. Greven, A. Kapitulnik, Phys. Rev. B 67, 014533 (2003)

6. W. D. Wise et al., Nat. Phys. 4, 696-699 (2008).

7. G. Ghiringhelli et al., Science 337, 821-825 (2012).

8. J. Chang et al., Nat. Phys. 8, 871-876 (2012).

9. S. Blanco-Canosa et al., Phys. Rev. Lett. 110, 187001 (2013).

10. T. P. Croft, C. Lester, M. S. Senn, A. Bombardi, S. M. Hayden, Phys. Rev. B 89, 224513 (2014).

11. E. H. da Silva Neto et al., Sci. Adv. 2, e1600782 (2016).

12. W. Tabis et al., Phys. Rev. B 96, 134510 (2017)

13. A. Perali, C. Castellani, C. Di Castro, M. Grilli, Phys. Rev. B Condens. Matter 54, 16216-16225 (1996)

14. S. A. Kivelson, E. Fradkin, V. J. Emery, Nature 393, 550-553 (1998).

15. L. E. Hayward, D. G. Hawthorn, R. G. Melko, S. Sachdev, Science 343, 1336-1339 (2014)

16. T. Wu et al., Nat. Commun. 6, 6438 (2015).

17. S. Gerber et al., Science 350, 949-952 (2015).

18. H. Jang et al., Proc. Natl. Acad. Sci. U.S.A. 113, 14645-14650 (2016).

19. J. Chang et al., Nat. Commun. 7, 11494 (2016)

20. F. Yu et al., Proc. Natl. Acad. Sci. U.S.A. 113, 12667-12672 (2016)

21. D. LeBoeuf et al., Nat. Phys. 9, 79-83 (2013).

22. T. Wu et al., Nature 477, 191-194 (2011).

23. L. Nie, L. E. H. Sierens, R. G. Melko, S. Sachdev, S. A. Kivelson, Phys. Rev. B 92, 174505 (2015)

24. Y. Caplan, G. Wachtel, D. Orgad, Phys. Rev. B 92, 224504 (2015).
25. S. Sadewasser, J. S. Schilling, A. P. Paulikas, B. W. Veal, Phys. Rev. B 61, 741-749 (2000).

26. S. M. Souliou et al., Phys. Rev. B 97, 020503 (2018).

27. C. Meingast et al., Phys. Rev. Lett. 67, 1634-1637 (1991).

28. U. Welp et al., Phys. Rev. Lett. 69, 2130-2133 (1992)

29. O. Kraut et al., Physica C 205, 139-146 (1993).

30. Materials and methods are available as supplementary materials.

31. C. W. Hicks et al., Science 344, 283-285 (2014),

32. C. W. Hicks, M. E. Barber, S. D. Edkins, D. O. Brodsky, A. P. Mackenzie, Rev. Sci. Instrum. 85, 065003 (2014).

33. A. Steppke et al., Strong peak in $T_{c}$ of $\mathrm{Sr}_{2} \mathrm{RuO}_{4}$ under uniaxial pressure. Science 355, eaaf9398 (2017).

34. M. Le Tacon et al., Nat. Phys. 10, 52-58 (2014).

35. E. M. Forgan et al., Nat. Commun. 6, 10064 (2015)

36. K.-P. Bohnen, R. Heid, M. Krauss, Europhys. Lett. 64, 104-110 (2003).

37. F. Weber et al., Phys. Rev. Lett. 107, 107403 (2011).

38. In presence of the oxygen-superstructure at this doping leve (Ortho-VIII), even more phonons can be expected (36 is the number of optical modes expected for the ortho-I structure of $\mathrm{YBa}_{2} \mathrm{Cu}_{3} \mathrm{O}_{7}$ ).

39. D. Reznik et al., Nature 440, 1170-1173 (2006).

40. D. Reznik, Physica C 481, 75-92 (2012).

41. M. Raichle et al., Phys. Rev. Lett. 107, 177004 (2011)

42. L. Chaix et al., Nat. Phys. 13, 952-956 (2017)

43. E. Kaneshita, M. Ichioka, K. Machida, Phys. Rev. Lett. 88, 115501 (2002)

44. L. Pintschovius et al., Phys. Rev. B 69, 214506 (2004).

45. Z. P. Yin, A. Kutepov, G. Kotliar, Phys. Rev. X 3, 021011 (2013).

46. J. A. Robertson, S. A. Kivelson, E. Fradkin, A. C. Fang A. Kapitulnik, Phys. Rev. B 74, 134507 (2006)

47. A. Del Maestro, B. Rosenow, S. Sachdev, Phys. Rev. B 74, 024520 (2006).

48. M. Le Tacon, Uniaxial pressure control of competing orders in a high temperature superconductor. Repository KITopen (2018); doi:10.5445/IR/1000086708

\section{ACKNOWLEDGMENTS}

We thank T. P. Devereaux, M.-H. Julien, S. A. Kivelson, D. Leboeuf, C. Meingast, J. Schmalian, and L. Taillefer for fruitful discussions, and FEI for assistance with the PFIB sample preparation. Funding: We acknowledge the support of the Helmholtz association through the program Science and Technology of Nanosystems and of the Max Planck Society. R.A.B. acknowledges partial financial support from Agencia Nacional de Promoción Científica y Tecnológica (PICT 2014 no. 2618) and CONICET (PIP 0446). Author contributions: IXS experiments were carried out by S.M.S., H.-H.K., E.L., M.M., M.T., A.B. and M.L.T. IXS data were analyzed by S.M.S. and M.L.T. X-ray diffraction experiments were carried out by H.-H.K., S.M.S., E. M.E. B., M.T M.M. G.G. C.W.H., and M.L.T. R.H. carried out the ab initio calculations. M.E.B., N.N., R.A.B., and C.W.H. performed the susceptibility measurements. Single crystals studied in this work were prepared by J.P., T.L., and S.M.S. PFIB preparation was carried out by P.M.M. and M.K. The strain devices used in this study were built by C.W.H., with assistance from M.L.T., H.-H.K., and E.L. S.M.S. and M.L.T. conceived the project. A.P.M., B.K., C.W.H., and M.L.T. supervised the project. M.L.T. and C.W.H. wrote the manuscript with input from all the coauthors. Competing interests: C.W.H. has $31 \%$ ownership of Razorbill Instruments, which has commercialized apparatus on which that used in this work are based. Data and materials availability: Raw data and simulation codes used for all figures in this paper and the supplementary materials are available at (48)

\section{SUPPLEMENTARY MATERIALS}

www.sciencemag.org/content/362/6418/1040/suppl/DC1 Materials and Methods

Supplementary Text

Figs. S1 to S9

References (49-55)

2 March 2018; accepted 25 October 2018

10.1126/science.aat4708 


\section{Science}

\section{Uniaxial pressure control of competing orders in a high-temperature superconductor}

H.-H. Kim, S. M. Souliou, M. E. Barber, E. Lefrançois, M. Minola, M. Tortora, R. Heid, N. Nandi, R. A. Borzi, G. Garbarino, A. Bosak, J. Porras, T. Loew, M. König, P. J. W. Moll, A. P. Mackenzie, B. Keimer, C. W. Hicks and M. Le Tacon

Science 362 (6418), 1040-1044.

DOI: $10.1126 /$ science.aat4708

\section{Squeezing into the third dimension}

Cuprate superconductors are known to harbor charge order in part of their phase diagram. Curiously, the order has a two-dimensional (2D) character at zero magnetic field, whereas a 3D order appears at high fields. Kim et al. now show that in a yttrium-based cuprate, a 3D charge order can be induced even at zero magnetic field. The authors compressed the material along one direction and measured a large inelastic $\mathrm{x}$-ray scattering signal that was consistent with the formation of a 3D order. The measurements suggest that the induced order is associated with an optical lattice mode in the material.

Science, this issue p. 1040

ARTICLE TOOLS

SUPPLEMENTARY MATERIALS

REFERENCES

PERMISSIONS http://science.sciencemag.org/content/362/6418/1040

http://science.sciencemag.org/content/suppl/2018/11/28/362.6418.1040.DC1

This article cites 55 articles, 8 of which you can access for free http://science.sciencemag.org/content/362/6418/1040\#BIBL

http://www.sciencemag.org/help/reprints-and-permissions

Use of this article is subject to the Terms of Service

Science (print ISSN 0036-8075; online ISSN 1095-9203) is published by the American Association for the Advancement of Science, $1200 \mathrm{New}$ York Avenue NW, Washington, DC 20005. The title Science is a registered trademark of AAAS.

Copyright $(2018$ The Authors, some rights reserved; exclusive licensee American Association for the Advancement of Science. No claim to original U.S. Government Works 